\title{
Correlation between the atlas morphology and the maxillo- mandibular divergence pattern
}

\author{
Hafiz Taha Mahmood \\ Aga Khan University, taha.mahmood@aku.edu \\ Mubassar Fida \\ Aga Khan University, mubassar.fida@aku.edu
}

Follow this and additional works at: https://ecommons.aku.edu/

pakistan_fhs_mc_surg_dent_oral_maxillofac

Part of the Dentistry Commons

\section{Recommended Citation}

Mahmood, H., Fida, M. (2018). Correlation between the atlas morphology and the maxillo-mandibular divergence pattern. Journal of the College of Physicians and Surgeons, 28(9), 690-694.

Available at: https://ecommons.aku.edu/pakistan_fhs_mc_surg_dent_oral_maxillofac/87 


\title{
Correlation between the Atlas Morphology and the Maxillo-mandibular Divergence Pattern
}

\author{
Hafiz Taha Mahmood and Mubassar Fida
}

\begin{abstract}
Objective: To evaluate the correlation between atlas morphology and maxillo-mandibular divergence.

Study Design: Cross-sectional, analytic study.

Place and Duration of Study: Dental Clinics, The Aga Khan University Hospital, Karachi, Pakistan from February to August 2017. Methodology: Pretreatment lateral cephalograms of 208 subjects, aged 18 to 25 years, were evaluated. The atlas parameters were categorized into atlas dorsum, anteroposterior and ventrum, and measured on View Pro-X software. Various maxillary (FPPP, SNPP and FHPP angles) and mandibular (SNGoGn, saddle, articulare, gonial, sum of posterior and $Y$-axis angles) parameters were used to evaluate the divergence pattern of the individuals. Mann-Whitney U-test was used to compare atlas and maxillo-mandibular parameters between genders. Spearman correlation was used to correlate atlas and maxillo-mandibular parameters across genders. A p-value $\leq 0.05$ was considered as statistically significant.

Results: Statistically significant differences were found between various atlas and maxillo-mandibular parameters between genders. With atlas dorsum, the saddle angle $(r=-0.3)$ in males; whereas gonial $(r=-0.2), Y$-axis $(r=0.1)$ and SNPP $(r=-0.2)$ angles in females showed significant weak correlation. With atlas anteroposterior, saddle $(r=-0.2)$, articulare $(r=0.2)$, SNPP $(r=-0.2)$ and FHPP $(r=-0.3)$ angles in males showed significant weak correlation. However, only the SNPP angle $(r=-0.2)$ in females showed a significant weak correlation with atlas ventrum.

Conclusion: A weak correlation was found between atlas parameters and various maxillo-mandibular angular parameters in both genders. Therefore, atlas morphology cannot be regarded as a good predictor of future maxillo-mandibular divergence pattern.
\end{abstract}

Key Words: Cervical atlas. Maxillofacial development. Vertical dimension.

\section{INTRODUCTION}

Craniofacial growth causes the jaw bones to grow in size and change their spatial relationship to the cranial base. This change in the orientation of the maxilla and mandible in the vertical plane is referred to as jaw rotations. Björk introduced growth rotations as a feature of normal facial growth. ${ }^{1,2}$ Jaw rotations may occur either in a forward or backward direction, but an average growth pattern usually depicts a moderate degree of forward growth rotation of both jaws. ${ }^{3}$ Various studies have used different terminologies for describing the maxillary and mandibular rotations. ${ }^{4-6}$

Jaw rotations during growth not only affect the final position of the facial bones but it may also affect the surrounding soft tissues and teeth. ${ }^{7,8}$ In forward rotators, there is more posterior growth than anterior and the individual is characterized by short face, short lower anterior facial height, horizontal palatal plane, decreased mandibular plane angle, deep bite and crowded

Department of Orthodontics Surgery, The Aga Khan University Hospital, Stadium Road, Karachi.

Correspondence: Dr. Hafiz Taha Mahmood, Resident Orthodontics, Section of Dentistry, Department of Surgery,

The Aga Khan University Hospital, P.O Box 3500, Stadium

Road, Karachi-74800.

E-mail:taha.mahmood@aku.edu

Received: January 23, 2018; Accepted: May 15, 2018. incisors. ${ }^{9}$ While in backward rotators, there is more anterior growth than posterior and the individual is characterized by long face, excessive lower anterior facial height, tipping of palatal plane down posteriorly and up anteriorly, increased mandibular plane angle, open bite and proclined incisors. 9 The treatment modality for the divergence pattern of the individual depends on the etiology, growth status, smile and lip line, incisor display and severity of the malocclusion. The treatment options include growth modification, camouflage, orthognathic surgery or the utilization of temporary anchorage devices. ${ }^{10}$

Atlas is the first cervical vertebra which connects the base of skull to the spine. It has a vital role in the growth and function of the craniofacial complex. The growth of the atlas is usually completed by 7 years of age, ${ }^{11}$ while the growth of the maxilla and mandible continues throughout the adolescence. ${ }^{6}$ If the morphology of the atlas and the maxillary and mandibular growth patterns are significantly correlated, it will be helpful in identifying the future vertical growth pattern of a child. The morphology of the atlas has been regarded as an indicator of the direction of mandibular growth. Huggare has shown that there is a significant relationship between the atlas dorsal arch and the direction of mandibular growth rotation. ${ }^{12}$ Nisayif and Al-Sahaf found significant correlation between the atlas dorsal arch and atlas anteroposterior and mandibular growth rotation. ${ }^{13}$ They reported that as the height of the atlas dorsal arch 
and atlas anteroposterior increases, there is increased horizontal rotation of the mandible. ${ }^{13}$

A survey of pertinent literature shows that currently no local study has been conducted on this topic and very few studies have reported the correlation of the morphology of the atlas with the mandibular rotation. ${ }^{12,13}$ Both maxilla and mandible contribute to the divergent pattern of an individual and none of the studies have yet reported the relationship of atlas morphology with the maxillary rotation. Therefore, this study was planned to determine the correlation between the atlas morphology and maxillo-mandibular divergence pattern.

\section{METHODOLOGY}

Subjects with ages between 18-25 years with goodquality standardized pretreatment lateral cephalograms were included in the study. Subjects with previous history of orthodontic or orthopedic treatment, presence of any craniofacial, dental anomaly, syndromes or history of trauma and surgery involving facial and vertebral structures were excluded from the study.

Data were collected retrospectively from the pretreatment lateral cephalograms of orthodontic patients visiting the Dental Clinics, The Aga Khan University Hospital, Karachi, Pakistan from February to August 2017. The sample size was calculated in NCSS PASS Software (Kaysville, UT, USA) using the correlation value $(r=-0.25)$ between the gonial angle and atlas anteroposterior as reported by Nisayif and Al-Sahaf. ${ }^{13}$ Keeping $\alpha=0.05$ and power of study as $80 \%$, this gave us a sample size of 189 subjects. This number was inflated by $10 \%$ to obtain a final sample size of 208 subjects (N). The entire sample consisted of equal number of male and female participants. An approval from the Ethical Review Committee was obtained prior to conducting the study.

Digital images of lateral cephalogram were evaluated and measured using View Pro-X (Rogan-Delft, Veenendaal, The Netherlands) software. The morphology of atlas vertebra was analyzed using the method as proposed by Huggare and Kylämarkula, ${ }^{14}$ and characterized into atlas dorsum, atlas ventrum and atlas anteroposterior (Figure 1).

For evaluation of divergence pattern of subjects, lateral cephalograms of all participants were traced manually by the principal investigator on an acetate paper in a dark room using an illuminator. The various mandibular angular parameters used were: sella nasion to gonion gnathion (SNGoGn) angle, sella nasion articulare (saddle) angle, sella articulare gonion (articulare) angle, articulare gonion menton (gonial) angle, the sum of posterior (saddle + articulare + gonial) angles and the Frankfort horizontal to sella gnathion (Y-axis) angle. The various maxillary angular parameters that we have used were: facial plane to palatal plane (FPPP) angle, sella nasion to palatal plane (SNPP) angle, and the Frankfort

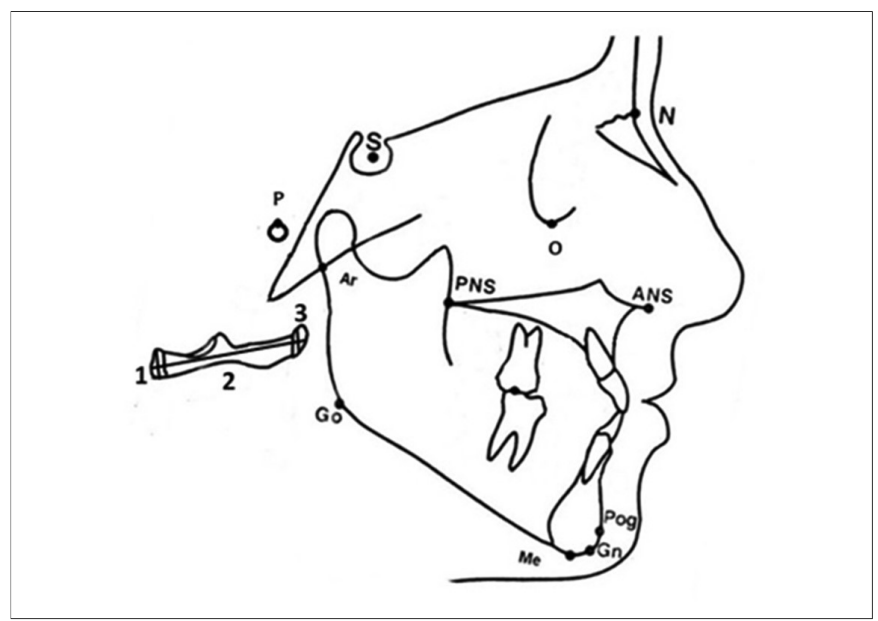

Figure 1: Evaluation of atlas morphology.

1: Atlas dorsum is the maximum vertical extent of the atlas dorsal arch.

2: Atlas anteroposterior is the maximum anteroposterior horizontal distance.

3: Atlas ventrum is the maximum vertical extent of the atlas ventral arch.

horizontal to palatal plane (FHPP) angle. The divergence pattern of an individual (normodivergent, hypodivergent or hyperdivergent) was based on the majority of above mentioned maxillo-mandibular angular parameters.

Data were analyzed in SPSS software for Windows (version 19.0, IBM, Armonk, NY). Kolmogorov-Smirnov test was used to check the normality of the measurements, which showed a non-normal distribution; hence, nonparametric tests were applied. Mann-Whitney U-test was used to compare maxillo-mandibular and atlas (atlas dorsum, ventrum and anteroposterior distance) parameters between genders. Spearman correlation was used to determine the correlation between maxillomandibular and atlas parameters in both sexes. A p-value of $\leq 0.05$ was considered as statistically significant.

To rule out the measurement error, 30 cephalograms were randomly selected, retraced and measurements were repeated by the principal investigator for evaluation of intra-examiner reliability. Intraclass correlation coefficient was applied which showed strong correlation between the two sets of readings (Table I).

\section{RESULTS}

Statistically significant differences were found between various maxillo-mandibular and atlas parameters when compared in males and females. Hence, further statistical analyses were performed separately for each gender (Table II).

The correlations between atlas dorsum and maxillomandibular parameters were evaluated for both sexes (Table III). Among the mandibular parameters, the saddle angle showed significant weak negative correlation $(r=-0.3, p=0.001)$ with atlas dorsum in males; whereas, the gonial angle showed significant weak negative correlation $(r=-0.2, p=0.01)$, while $Y$-axis angle showed significant weak positive $(r=0.1, p=0.04)$ correlation with atlas dorsum in females. Among the 
maxillary parameters, only SNPP angle in females showed significant weak negative correlation $(r=-0.2$, $p=0.02$ ) with atlas dorsum.

Table I: Assessment of the reliability of measurements.

\begin{tabular}{l|c|c|c}
\hline \multirow{2}{*}{ Parameters } & \multicolumn{2}{|c|}{ Mean \pm SD } & \multirow{2}{*}{ ICC } \\
\cline { 2 - 3 } & First & Second & \\
\hline Mandibular & & & \\
SNGoGn angle (degree) & $32.0 \pm 4.4$ & $32.7 \pm 4.3$ & 0.9 \\
Saddle angle (degree) & $126.8 \pm 6.4$ & $127.2 \pm 6.4$ & 0.8 \\
Articulare angle (degree) & $141.2 \pm 6.2$ & $141.8 \pm 6.2$ & 0.9 \\
Gonial angle (degree) & $127.0 \pm 7.4$ & $128.2 \pm 8.4$ & 0.9 \\
Sum of posterior angles (degree) & $395.2 \pm 5.8$ & $395.7 \pm 5.8$ & 0.8 \\
Y-axis angle (degree) & $60.9 \pm 3.7$ & $61.3 \pm 3.3$ & 0.9 \\
\hline Maxillary & & & \\
FPPP angle (degree) & $86.7 \pm 3.9$ & $87.2 \pm 4.0$ & 0.8 \\
SNPP angle (degree) & $7.9 \pm 4.0$ & $8.4 \pm 4.0$ & 0.8 \\
FHPP angle (degree) & $0.1 \pm 2.2$ & $0.6 \pm 2.3$ & 0.8 \\
\hline Atlas & & & \\
Dorsum (mm) & $10.9 \pm 1.8$ & $8.4 \pm 4.0$ & 0.7 \\
Anteroposterior (mm) & $50.6 \pm 3.5$ & $51.5 \pm 3.8$ & 0.8 \\
Ventrum (mm) & $10.9 \pm 1.5$ & $11.5 \pm 2.5$ & 0.8 \\
\hline$n=30 ; \quad I C C=$ Intraclass correlation coefficient & &
\end{tabular}

Table II: Comparison of maxillo-mandibular parameters and atlas morphology between genders.

\begin{tabular}{l|c|c|c}
\hline Parameters & $\begin{array}{c}\text { Males }(\mathrm{n}=104) \\
\text { Median (IQR) }\end{array}$ & $\begin{array}{c}\text { Females (n=104) } \\
\text { Median (IQR) }\end{array}$ & p-value \\
\hline Mandibular & $29.0(8.0)$ & $31.5(6.0)$ & $0.001^{* *}$ \\
SNGoGn angle & $122.5(9.9)$ & $125.5(6.0)$ & $0.046^{*}$ \\
Saddle angle & $142.25(8.0)$ & $142.5(9.4)$ & 0.729 \\
Articulare angle & $123.0(10.5)$ & $126.25(7.8)$ & $0.006^{*}$ \\
Gonial angle & $391.0(9.5)$ & $394.25(8.3)$ & $0.001^{* *}$ \\
Sum of posterior angles & $61.0(4.0)$ & $60.0(5.0)$ & 0.770 \\
Y-axis angle & & & \\
\hline Maxillary & $88.0(5.8)$ & $88.0(5.0)$ & 0.342 \\
FPPP angle & $7.0(5.0)$ & $8.0(4.5)$ & $0.036^{*}$ \\
SNPP angle & $0.50(5.0)$ & $0.5(3.9)$ & 0.945 \\
FHPP angle & $10.1(2.4)$ & $9.9(2.0)$ & 0.40 \\
\hline Atlas & $50.5(3.7)$ & $46.6(3.5)$ & $0.001^{* *}$ \\
Dorsum & $10.8(1.7)$ & $10.1(1.7)$ & $0.001^{* *}$ \\
Anteroposterior & \multicolumn{3}{|l}{} \\
Ventrum & $n=208 ;$ IQR $=$ Inter Quartile Range; Mann Whitney U-test \\
${ }^{*} p<0.05 ;{ }^{*} p<0.001$. &
\end{tabular}

Spearman correlation was used to determine correlation between atlas anteroposterior and maxillo-mandibular parameters in both sexes (Table III). Among the mandibular parameters, the saddle angle showed significant weak negative $(r=-0.2, p=0.03)$, while articulare angle showed significant weak positive correlation $(r=0.2, p=0.005)$ with altas anteroposterior in males. Among the maxillary parameters, SNPP $(r=-0.2$, $p=0.003)$ and FHPP $(r=-0.3, p=<0.001)$ angles in males showed significant weak negative correlation with atlas anteroposterior. However, none of the maxillomandibular parameters in females showed significant correlation with atlas anteroposterior.

Similarly, the correlation between atlas ventrum and maxillo-mandibular parameters was obtained for both genders (Table III). The only SNPP angle in females showed significant weak negative correlation $(r=-0.2$, $p=0.03$ ) with atlas ventrum.

\section{DISCUSSION}

Prediction of anticipated maxillo-mandibular divergence pattern of the individual has long been debated in the orthodontic literature. Björk proposed seven structural signs to predict the type of growth rotations. ${ }^{15}$ As the number of variables present in a certain individual increases, the likelihood of an accurate prediction of divergence pattern also increases. Skieller et al. have reported that these variables are only applicable in cases with severe skeletal discrepancy. ${ }^{16}$ Another method was proposed by Skieller et al., 16 which is based on evaluation of mandibular inclination, intermolar angle, shape of lower border of mandible and inclination of symphysis on lateral cephalogram. However, Leslie et al. have reported that the information obtained using this method is inappropriate and cannot be used to predict the direction of future divergence pattern of the individual. ${ }^{17}$ Recently, Yasa et al. have found significant association between the sella turcica morphology and the divergence pattern. ${ }^{18}$

The cervical spine, particularly the first and second vertebrae, are adjacent to the mandible and studies

Table III: Correlation between atlas dorsum, anteroposterior and ventrum and maxillo-mandibular parameters in males and females.

\begin{tabular}{|c|c|c|c|c|c|c|c|c|c|c|c|c|}
\hline \multirow[t]{3}{*}{ Parameters } & \multicolumn{4}{|c|}{ Atlas Dorsum } & \multicolumn{4}{|c|}{ Atlas Anteroposterior } & \multicolumn{4}{|c|}{ Atlas Ventrum } \\
\hline & \multicolumn{2}{|c|}{ Males } & \multicolumn{2}{|c|}{ Females } & \multicolumn{2}{|c|}{ Males } & \multicolumn{2}{|c|}{ Females } & \multicolumn{2}{|c|}{ Males } & \multicolumn{2}{|c|}{ Females } \\
\hline & r-value & $p$-value & $r$-value & $p$-value & $r$-value & $\mathrm{p}$-value & r-value & $p$-value & r-value & $p$-value & r-value & $p$-value \\
\hline \multicolumn{13}{|l|}{ Mandibular } \\
\hline SNGoGn angle & -0.177 & 0.072 & -0.134 & 0.175 & -0.142 & 0.150 & 0.037 & 0.712 & 0.007 & 0.947 & -0.085 & 0.392 \\
\hline Saddle angle & -0.321 & $0.001^{*}$ & 0.051 & 0.609 & -0.208 & $0.034^{*}$ & 0.051 & 0.606 & -0.175 & 0.075 & -0.007 & 0.947 \\
\hline Articulare angle & 0.144 & 0.146 & 0.110 & 0.265 & 0.274 & $0.005^{\star}$ & 0.091 & 0.358 & 0.081 & 0.414 & 0.126 & 0.203 \\
\hline Gonial angle & 0.029 & 0.772 & -0.245 & $0.012^{*}$ & -0.124 & 0.208 & -0.061 & 0.537 & 0.092 & 0.354 & -0.108 & 0.277 \\
\hline Sum of posterior & -0.142 & 0.149 & -0.048 & 0.628 & -0.145 & 0.143 & 0.028 & 0.777 & -0.031 & 0.756 & 0.020 & 0.840 \\
\hline Y-axis angle & 0.062 & 0.534 & 0.197 & $0.045^{*}$ & 0.126 & 0.203 & 0.086 & 0.384 & 0.132 & 0.182 & 0.169 & 0.087 \\
\hline \multicolumn{13}{|l|}{ Maxillary } \\
\hline FPPP angle & -0.042 & 0.670 & -0.176 & 0.074 & -0.154 & 0.118 & -0.135 & 0.172 & 0.016 & 0.868 & -0.178 & 0.071 \\
\hline SNPP angle & -0.182 & 0.064 & -0.222 & $0.024^{*}$ & -0.290 & $0.003^{*}$ & -0.086 & 0.387 & -0.117 & 0.235 & -0.203 & $0.038^{*}$ \\
\hline FHPP angle & -0.145 & 0.141 & -0.155 & 0.115 & -0.343 & $<0.001^{\star *}$ & -0.125 & 0.207 & -0.139 & 0.160 & -0.067 & 0.502 \\
\hline
\end{tabular}

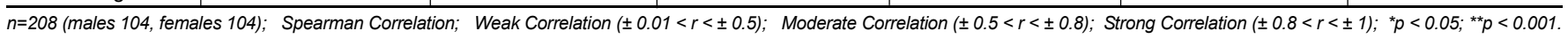


have reported their association with the size of the mandible, mandibular divergence and craniofacial morphology.19-21 Hence, the aim of the present study was to determine the correlation between atlas dorsum, atlas ventrum and atlas anteroposterior and the maxillomandibular divergence pattern of the individual.

Huggare was the first to assess atlas morphology and regarded it as the predictor of mandibular growth rotation. ${ }^{12} \mathrm{He}$ reported that the height of atlas dorsal arch significantly correlated with the horizontal rotation of the mandible. The atlas as seen on lateral cephalogram was characterized into atlas dorsum, atlas ventrum and atlas anteroposterior. The authors used various maxillo-mandibular parameters for categorization of individuals into normo, hyper or hypodivergent pattern in the present study. However, only a weak correlation of atlas dorsum was found with mandibular parameters.

In this study, statistically significant differences were found between atlas (atlas anteroposterior and ventrum) and various maxillo-mandibular (SNPP, SNGoGn, saddle, gonial and sum of posterior angles) parameters across gender. Nisayif and Al-Sahaf found statistically significant difference in the atlas anteroposterior only in both genders. ${ }^{13}$ Gender differences were also found in the studies by Kylamarkula and Huggare and Al-Hashimi and Al-Azawi. ${ }^{22,23}$

This study reveals varying correlation between atlas and maxillary and mandibular parameters, which are stratified according to the gender. Nisayif and Al-Sahaf conducted a study on Iraqi population with a sample size of 200 individuals and evaluated correlation between atlas parameters and various mandibular parameters only. ${ }^{13}$ Further, they did not stratify their results according to gender. They have reported significant weak negative correlation between SNGoGn, sum of posterior angles, gonial angle and Y-axis, and atlas dorsal arch. Similarly, the present authors also found significant weak negative correlation between gonial angle and atlas dorsum in the female sample; on the contrary, there was a significant weak positive correlation between $\mathrm{Y}$-axis angle and atlas dorsum in females. They earlier also reported a significant weak negative correlation between SNGoGn, sum of posterior, gonial and $\mathrm{Y}$-axis angles, and atlas anteroposterior. ${ }^{13}$ However, the present authors found a significant weak negative correlation of saddle angle and a significant weak positive correlation of articulare angle with atlas anteroposterior in the male sample. Additionally, they have reported non-significant weak correlation between atlas ventrum and various mandibular angular parameters. ${ }^{13}$ Similarly, the present authors also found non-significant weak correlation between atlas ventrum and various mandibular angular parameters in both genders. The differences in the results could be due to morphogenetic differences in different population and manual tracing of the lateral cephalograms and measurements of angular parameters in this study.

Another study conducted by Jahjah and Hassan in Caucasian population with a sample size of 30 individuals, found weak positive correlation between saddle and gonial angles and atlas anteroposterior and moderate negative correlation between articulare and sum of posterior angles in male participants. ${ }^{24}$ Whereas, the present work found weak negative correlation between saddle, gonial and sum of posterior angles and atlas anteroposterior and a weak positive correlation between articulare angle and atlas anteroposterior in male sample. Moreover, Jahjah et al. reported a weak negative correlation between saddle angle and atlas anteroposterior and weak positive correlation between articulare, gonial and sum of posterior angles and atlas anteroposterior in female participants. ${ }^{24}$ On the contrary, the present authors found moderate positive correlation between saddle, gonial and sum of posterior angles and a weak positive correlation between articulare angle and atlas anteroposterior in female sample. The differences in the results could be due to CBCT scan based lateral cephalogram used in their study which gives high quality images with less magnification error. ${ }^{25}$

The authors have found a weak correlation between atlas morphology and maxillo-mandibular divergence pattern, so predicting the maxillo-mandibular divergence pattern using the atlas morphology is clearly not indicated, and other means of predicting future growth need to be identified. Longitudinal study design is necessary to evaluate the growth status of an individual, while we have acquired the data cross-sectionally which has certain limitations. A single centered study with manual tracing and measurements of angular parameters on $2 \mathrm{D}$ imaging modality are the limitations of the study. Moreover, only the sagittal and vertical measurements can be performed on lateral cephalogram for atlas dimensions, so we recommend a three dimensional or volumetric assessment of atlas morphology to predict the future divergence pattern of the individual.

\section{CONCLUSION}

Statistically significant weak correlation was found between atlas and maxillo-mandibular parameters in both genders. Therefore, longitudinal studies are needed to evaluate the divergence pattern using atlas morphology.

\section{REFERENCES}

1. Björk A. Variations in the growth pattern of the human mandible: longitudinal radiographic study by the implant method. J Dent Res 1963; 42:400-11.

2. Björk A. The use of metallic implants in the study of facial growth in children: method and application. Am J Phys Anthropol 1968; 29:243-54. 
3. Björk A, Skiellar V. Normal and abnormal growth of the mandible. A synthesis of longitudinal cephalometric implant studies over a period of 25 years. Eur J Orthod 1983; 5:1-46.

4. Björk A, Skieller V. Facial development and tooth eruption. An implant study at the age of puberty. Am J Orthod 1972; 62:9-83.

5. Schudy FF. The rotation of the mandible resulting from growth: its implications in orthodontic treatment. Angle Orthod 1965; 35:36-50.

6. Solow B, Houston WJ. Mandibular rotations: concepts and terminology. Eur J Orthod 1988; 10:177-9.

7. Bahrou S, Hassan AA, Khalil F. Facial proportions in different mandibular rotations in class I individuals. Int Arab $J$ Dent 2014; 5:9-18.

8. Sayagh NM, Saleem NR, Abdul-Qadir MY. Analysis of soft tissue facial profile in different vertical growth patterns. Al-Rafidain Dent J 2011; 11:346-56.

9. Profitt WR, Fields HW, Sarver DM. Contemporary orthodontics. 5th ed. St. Louis: Mosby; 2013.

10. Graber LW, Vanarsdall RL, Vig KWL. Orthodontics, Current principles and techniques. 5th ed. Canada: Mosby; 2012.

11. Fesmire FM, Luten RC. The pediatric cervical spine: developmental anatomy and clinical aspects. J Emerg Med 1989; 7:133-42.

12. Huggare J. The first cervical vertebra as an indicator of mandibular growth. Eur J Orthod 1989; 11:10-6.

13. Nisayif $\mathrm{DH}, \mathrm{Al}-\mathrm{Sahaf} \mathrm{NH}$. The relationship between the morphology of the first cervical vertebra and the direction of mandibular rotation in Iraqi adults. Iraqi Orthod J 2005; 1:32-5.

14. Huggare J, Kylämarkula $S$. Morphology of the first cervical vertebra in children with enlarged adenoids. Eur J Orthod 1985; 7:93-6.
15. Björk A. Prediction of mandibular growth rotation. Am J Orthod 1969; 55:585-99.

16. Skieller V, Björk A, Linde-Hansen T. Prediction of mandibular growth rotation evaluated from a longitudinal implant sample. Am J Orthod 1984; 86:359-70.

17. Leslie LR, Southard TE, Southard KA, Casko JS, Jakobsen JR, Tolley EA, et al. Prediction of mandibular growth rotation: assessment of the Skieller, Björk, and Linde-Hansen method. Am J Orthod Dentofacial Orthop 1998; 114:659-67.

18. Yasa Y, Buyuk SK, Benkli YA, Arslann A, Topbasi NM. The size and shape of the sella turcica in adolescent orthodontic patients with different vertical growth patterns. Clin Dent Res 2017; 41:3-9.

19. Schafer RC. Clinical biomechanics: musculoskeletal actions and reactions. 2nd Ed, Baltimore. Williams and Wilkins; 1987.

20. Springate SD. A re-investigation of the relationship between head posture and craniofacial growth. Eur J Orthod 2012; 34: 397-409.

21. Festa F, Tecco S, Dolci M, Ciufolo F, Di Meo S, Filippi MR, et al. Relationship between cervical lordosis and facial morphology in Casucasian women with skeletal class II malocclusion: a cross-sectional study. Cranio. 2003; 21:121-9.

22. Kylämarkula $S$, Huggare J. Head posture and the morphology of the first cervical vertebra. Eur J Orthod 1985; 7:151-6.

23. Al-Hashimi HAH, Al-Azawi ZZ. Association of the morphology of the atlas vertebra with the morphology of the mandible. Mustansiria Dent J 2008; 5:194-9.

24. Jahjah $Y$, Hassan $H$. Dimensions of atlas and its relation with jaws rotation (CBCT scan study). Tish Uni J Res Sci Stud 2015; 37:333-56.

25. Sukovic P. Cone beam computed tomography in craniofacial imaging. Orthod Craniofac Res 2003; 6:31-6. …두..... 